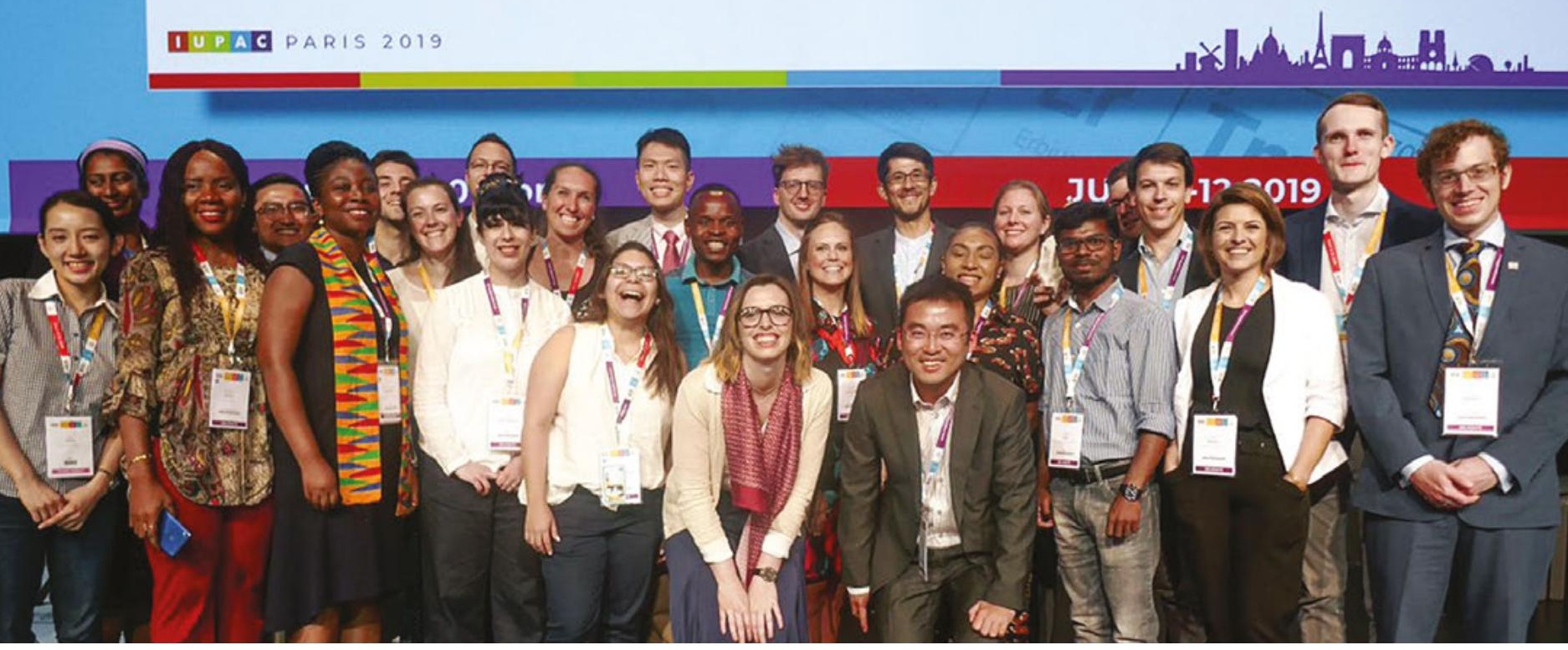

special guest speakers. The webinars will take on a variety of formats depending on the topic of discussion, including traditional presentations, roundtables, and $Q \& A$ discussions. The webinars will feature broad topics such as "Building your science brand," "Finding your voice at work," and "Sharing your voice with the world."

Through the ChemVoices program, we are hoping to increase the engagement and visibility of those who were awarded an element. More importantly, participation in this program will help to cultivate the professional and personal development of the PTChemist awardees, develop programming that is relevant and of interest to early-career researchers internationally, and provide an opportunity to cross-promote other IUPAC and IYCN activities to an audience that may be outside our combined reach.

\section{Digital Representation of Units of Measure}

The current state of the digital representation of units of measure across domains is a significant problem relative to the interoperability of data. Across the scientific disciplines there is a wide variety of knowledge about, focus on, and care with the recording of a unit of measure with each piece of experimental, calculated, modeled or derived data. Much information is available for annotation of units for humans, however there is no authoritative source for how to represent and store units of measures (in any units' system) in digital systems. This is a fundamental problem for data science currently and a major problem for the future integration of large, heterogeneous datasets both within and across disciplines. It is the most important single issue for the development of general or domain repositories, for the ideas behind Big Data and Open Data and the implementation of systems that support Findable, Accessible, Interoperable, and Reusable (FAIR) data.
To finalize the first phase of ChemVoices, an event at IUPAC2021 will be held to celebrate the PTChemists and all of those who participated in the webinars. Whilst still in the early stages of our planning, we are additionally working to enhance the programming and opportunities for early-career researchers at IUPAC2021, with this event being a key piece in that effort.

We are excited to bring ChemVoices to life, and we encourage you all to visit the website: www.ChemVoices. org to learn more about the team behind the webinars, and to register for our next event!

For more information and comments, contact Task Group Chair Lori Ferrins $<$ l.ferrins@northeastern.edu>| https://iupac.org/project/2020-012-2-020 | https://chemvoices.org/

The issue is the topic of a current CODATA project-called DRUM-co-chaired by Robert Hanisch (NIST, USA), Jeremy Frey (University of Southampton, UK | IUPAC Commission I.1), Simon Cox (CSIRO, Australia), and Stuart Chalk (University of North Florida, USA | IUPAC CPCDS)

As reported by Simon Hodson during the CODATA virtual GA in June 2020, the DRUM task group is preparing a position paper that will make the case for the importance of digitals units of measure and invite the presentation of uses cases that demonstrate the utility and importance of digital representation of units of measures and/or illustrate pain points.

This project will also propose liaison with BIPM in connection with their Digital SI workshop, tentatively announced for 25-26 Feb 2021. (https://www.bipm.org/ en/conference-centre/bipm-workshops/digital-si/)

For more information and comments, contact Jeremy Frey <j.g.frey@soton. ac.uk> | https://codata.org/initiatives/task-groups/drum/ 